\title{
Comunicación
}

\section{Schistosomus reflexus en un felino: reporte de caso}

\author{
Schistosomus reflexus in a feline: case report
}

\author{
A. Patiño, J.C. Aguirre ${ }^{1,2}$, R.S. Gallego ${ }^{1,3}$, R. Zambrano ${ }^{1,3}$, J.A. Buitrago ${ }^{1}$
}

\section{Resumien}

Se presenta el caso de una hembra felina de raza mestiza de 5 años en su primer parto llevada a consulta. La gata tenía un mortinato presente en el canal de parto el cual se extrae manualmente. Un segundo cachorro es expulsado vivo, pero por su la inviabilidad clínica se procedió a la eutanasia. Las dos crías presentaron una malformación en la región abdominal con exposición de algunas vísceras, considerada como el síndrome de Schistosomus reflexus. Se realizó la evaluación diagnóstica radiológica y por necropsia del segundo cachorro encontrando un defecto de cierre de la línea media abdominal con exposición de vísceras (intestino delgado, hígado, riñón derecho y bazo entre otros). En la evaluación radiológica se evidenció eventración de órganos abdominales sin diferenciación clara entre ellos, cifosis en el segmento toracolumbar, lordosis en la porción lumbar y oligodactilia en el miembro posterior derecho.

Palabras clave: anomalía congénita; felino; radiología; Schistosomus reflexus

\section{AbSTRaCT}

The case of a 5-year-old crossbred female cat in her first gestation was presented for consultation. The cat had a stillborn present in the birth canal, which was extracted manually. A second puppy is expelled alive but was euthanized because of her clinical infeasibility. The two puppies presented a malformation in the abdominal region with exposure of some viscera, considered as Schistosomus reflexus syndrome. The radiological and necropsy diagnostic evaluation of the second puppy was performed, finding a closure defect of the abdominal midline with viscera exposure (small intestine,

\footnotetext{
${ }^{1}$ Corporación Universitaria Remington, Facultad de Medicina Veterinaria, Grupo de Investigación GINVER, Medellín, Colombia

${ }^{2}$ Unidad Forense Veterinaria, Corporación Universitaria Remington, Medellín, Colombia

${ }^{3}$ Grupo de Imagenología Forense Veterinaria (IFOVET), Corporación Universitaria Remington, Medellin, Colombia
} 
liver, right kidney and spleen among others). The radiological evaluation showed the eventration of abdominal organs without clear differentiation between them, kyphosis in the thoracolumbar segment, lordosis in the lumbar portion and oligodactyly in the right posterior limb.

Key words: congenital anomaly; feline; radiology; Schistosomus reflexus

\section{INTRODUCCIÓN}

Schistosomus reflexus (SR) es una anomalía congénita del desarrollo embrionario incompatible con la vida que se presenta de manera ocasional. Se caracteriza por la exposición de vísceras abdominales, ocasionalmente torácicas, debido a un defecto extremo que ocasiona la fusión incompleta de la pared abdominal, asociada a una marcada desviación ventral de columna vertebral (Ferreira et al., 2013; Jana D y Jana M, 2013; Ahuja et al., 2017), que puede estar acompañada de malformación de extremidades, hipoplasia diafragmática y hepática, cambios genitourinarios y gastrointestinales (Laughton et al., 2005; Prestes y Megid, 2010; Ferreira et al., 2013; Leal et al., 2017) y, que por lo general, se presenta como un problema obstétrico en especies mayores (Tsuma y Abuom, 2008).

En Colombia, esta alteración del desarrollo solo ha sido reportada en bovinos (Gómez et al., 2018) y caninos (Molina et al., 2012), siendo este el primer caso reportado en felinos.

\section{Descripción del Caso}

Se presenta a consulta una hembra felina de raza mestiza de 5 años en su primer parto y con un cachorro presente en el canal de parto. El propietario desconoce el tiempo que lleva en esta condición.
$\mathrm{Al}$ examen físico general, la paciente se encuentra tranquila y estable con la mucosa oral rosada, levemente pálida y con los parámetros fisiológicos dentro de los rangos normales. El feto se encuentra en el canal del parto, se evidencia seco y se encuentra por fuera de su saco amniótico. El feto, que se encuentra en presentación longitudinal posterior, con posición dorso-sacra y actitud de extensión de los miembros posteriores, sale fácilmente por vía transvaginal después de realizarse una tracción. Una vez exteriorizado se evidencia que el cachorro mortinato presenta una malformación en la región abdominal con exposición de algunas vísceras cubiertas por una membrana delgada, aparentemente el peritoneo (Figura 1a).

Se administró a la madre una solución de leche deslactosada con azúcares, vía oral, la cual fue consumida ávidamente, después de lo cual la gata se tornó más dinámica y buscó huir del lugar en donde se encontraba. Luego de 40 minutos es expulsado un segundo cachorro vivo, por fuera de la membrana amniótica, y que presentó, al igual que el primer cachorro, una malformación abdominal con eventración de órganos abdominales (Figura $1 b)$. Se le realizó despeje de vías aéreas, secado y estimulación mecánica a la cual respondió de manera adecuada. El cachorro se observó estable, pero con reflejo de succión ausente. Por decisión del propietario se procedió a realizar la eutanasia con cloruro de potasio intracardiaco dos horas después de su nacimiento. Este cachorro fue remitido a la Unidad Forense Veterinaria de la Corporación Universitaria Remington para su estudio. 


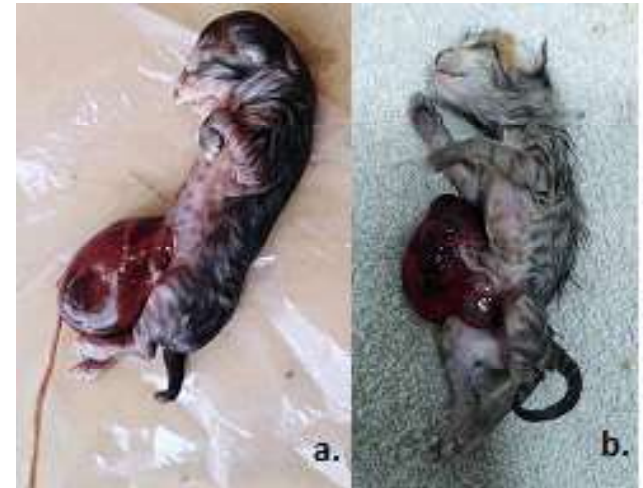

Figura 1. Cachorros nacidos con signos de Schistosomus reflexus. a. Primer cachorro de la camada (descartado por el médico tratante). b. Segundo cachorro de la camada remitido a la Unidad Forense de la Corporación Universitaria Remington

Después del nacimiento del segundo cachorro se determinó que la gata presentaba agalactia. Se le colocó una vía endovenosa y se le administraron fluidos intravenosos y fue dejada en observación. A los 30 minutos del nacimiento del segundo cachorro expulsó un tercer cachorro por fuera de su saco amniótico, morfológicamente normal, aunque de talla aparentemente pequeña, pero viable. Una hora después no hubo expulsión de membranas fetales ni de otros cachorros, por lo que se realizó palpación abdominal y se determinó que había otro cachorro en útero, además de otro contenido no identificado, por lo que se realizó una ecografía confirmando la presencia de otro cachorro y de algunas membranas fetales. Se aplicó oxitocina (2 UI/ $\mathrm{kg}$ ), expulsando un cuarto cachorro 90 minutos después del tercero, el cual nació dentro del saco amniótico, pequeño en talla, morfológicamente normal y completamente viable. Una hora después del cuarto cachorro fueron expulsadas las membranas fetales.

\section{Reporte Anatomo-Patológico}

Se presenta al servicio forense de la Corporación Universitaria Remington el cuerpo de un felino hembra neonato con un de- fecto de cierre de la línea media abdominal de $18 \mathrm{~mm}$ largo, $13 \mathrm{~mm}$ caudal al cartílago xifoides, con ancho de $12 \mathrm{~mm}$ y exposición de vísceras incluyendo al interior de la bolsa peritoneal extracorpórea los siguientes segmentos anatómicos: intestino delgado, hígado, riñón derecho (con signos congestivos), páncreas, bazo, colon mayor, colon descendente distendido y con presencia de meconio, con una correcta terminación de recto y esfínter anal. Las vísceras eventradas guardan forma y tamaño adecuado para su estadio gestacional (Figura 2).

El riñón izquierdo fue encontrado en la región sublumbar en posición adecuada, sin cambios externos anormales. En cavidad torácica, el diafragma se encuentra completo, cerrado y sin alteraciones, corazón en posición mediastínica, y pulmones bien situados y de apariencia enfisematosa; la tráquea se encuentra colapsada. Se nota deformidad en la columna vertebral tipo escoliosis hacia lateral derecho sobre segmento lumbosacro con deformidad ventral concomitante tipo lordosis inmediatamente caudal a la unión toracolumbar. Así mismo, sobre su miembro posterior derecho se percibe oligodactilia (didactilia) (Figura 3) con presencia de tres huesos metatarsianos sin presencia de artrogriposis. Los elementos del cordón umbilical ingresan de manera separada a la cavidad abdominal adherido al margen abdominal derecho.

\section{Estudio RadiolóGico}

Se realiza un estudio radiológico lateral derecho y ventro-dorsal (VET-RAY VR-40, USA), donde se aprecia eventración de órganos abdominales sin diferenciación clara entre ellos, lográndose visualizar asas de intestino delgado. En la vista ventro-dorsal se observan dos órganos radio-opacos compatibles con hígado y bazo. No se diferencian líneas diafragmáticas y se denota aumento de la cavidad torácica con disminución de la radiolucidez de los campos pulmonares. En ninguna de las tomas es posible delimitar la 


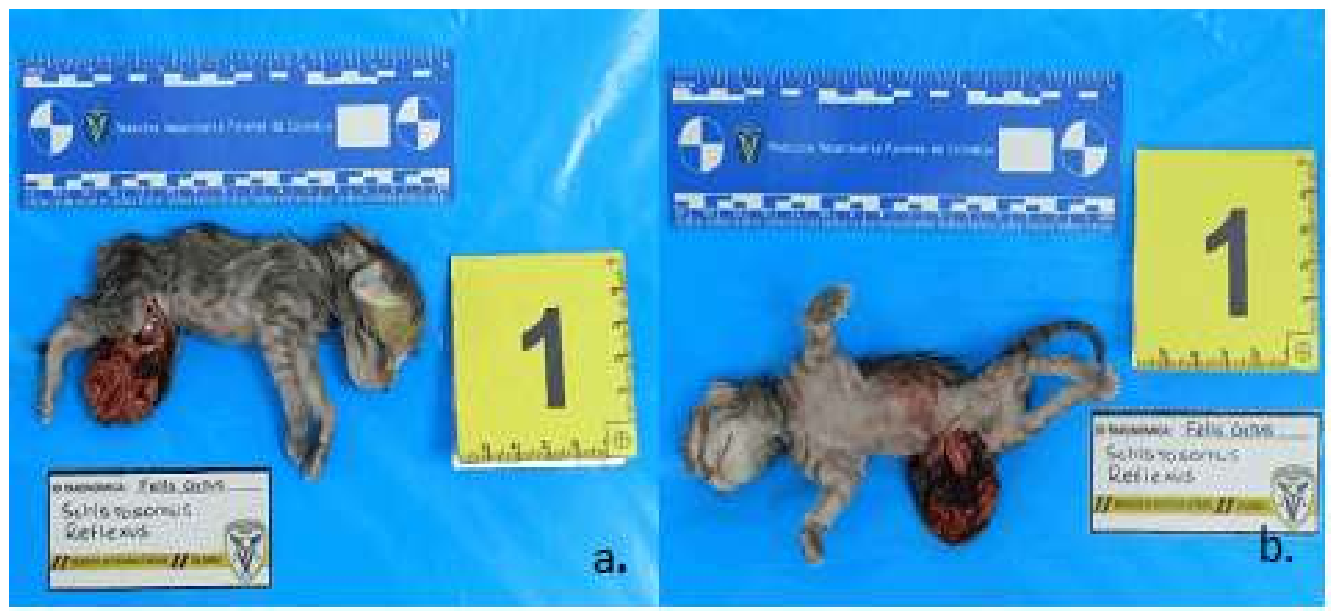

Figura 2. Felino neonato con defecto de cierre de la línea media abdominal, exposición de vísceras dentro de la bolsa peritoneal extracorpórea y deformidad en la columna vertebral. a. decúbito lateral izquierdo. b. decúbito dorsal

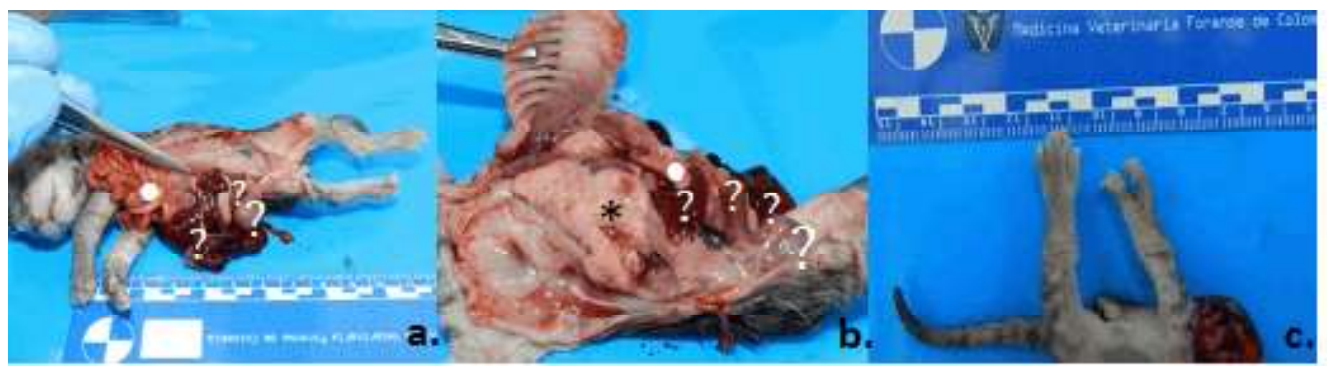

Figura 3. Felino hembra neonato con signos de Schistosomus reflexus. a. defecto de cierre de la línea media abdominal y exposición de vísceras: porción de intestino delgado $(\bullet)$, hígado ( $\bigcirc)$, porción de intestino grueso $(\square)$, riñón derecho $(\square)$; b. pulmón con aspecto enfisematoso $(*)$, bazo $(\square)$, segmentos anatómicos; c. oligodactilia en miembro posterior derecho

silueta cardiaca, y en la vista ventro-dorsal se observa desviación del eje cardiaco hacia el hemitórax derecho.

En la vista lateral derecha, se percibe a nivel de columna, en el segmento toraco-lumbar, una tendencia a la cifosis, mientras que en el segmento lumbar se aprecia marcada lordosis con cambios morfológicos de los cuerpos vertebrales (Figura 4a). En la vista ventro- dorsal se confirman cambios morfológicos de vértebras lumbares y desviación lateral derecha (escoliosis) ocasionando desviación de los huesos de la cadera; en el saco extracorpóreo se observa sobreposición de vísceras abdominales. En el miembro posterior derecho se aprecia oligodactilia con presencia de tres metatarsianos y dos dígitos (Figura 4b). 


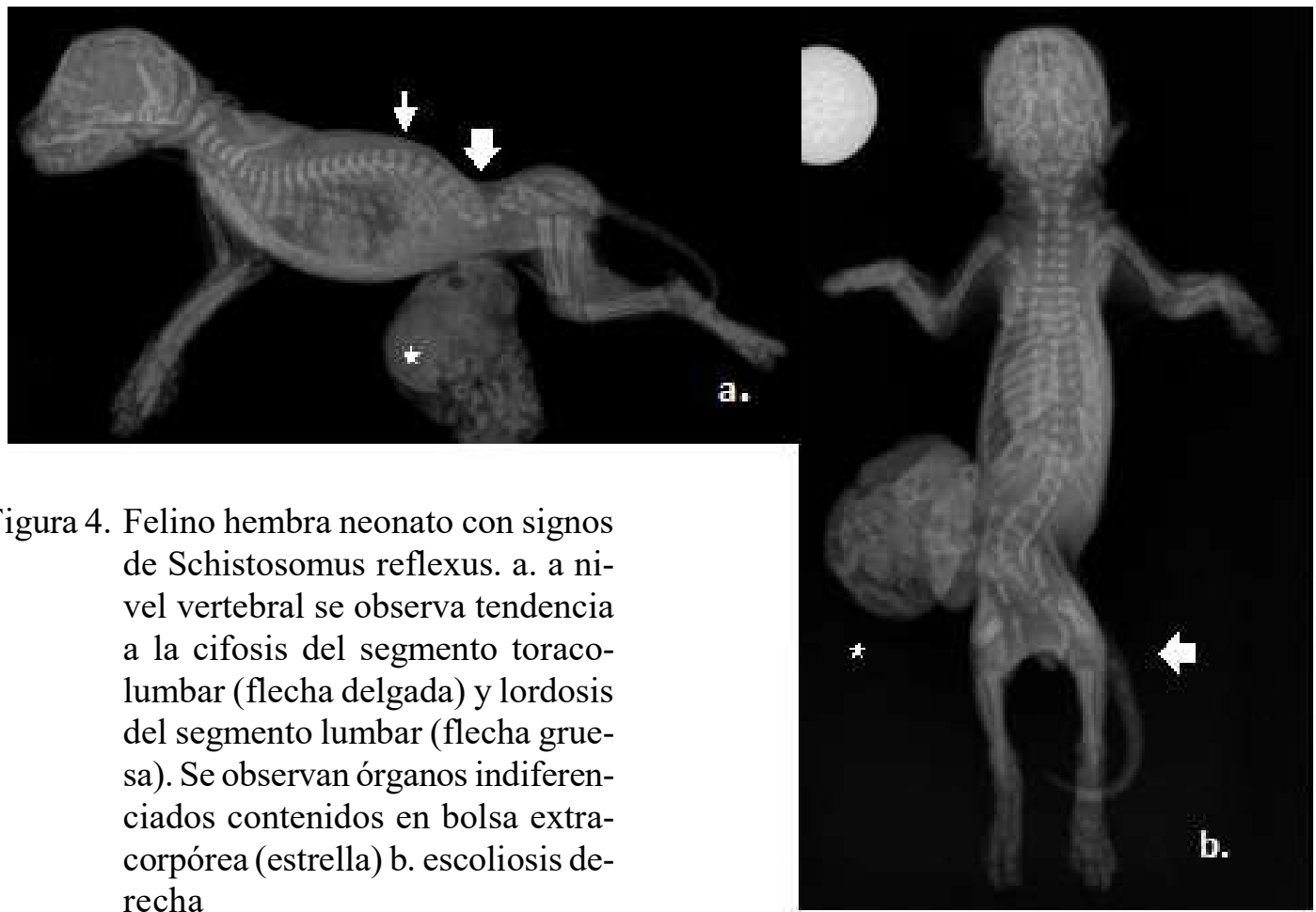

\section{Discusión}

El SR se define como un feto con malformaciones congénitas o anomalías de desarrollo tan pronunciadas que suelen ser grotescas y generalmente inviables (Batra et al., 2015), usualmente ocasionadas por anomalías del desarrollo embrionario, ya sea por factores genéticos o ambientales que afectan drásticamente los procesos de desarrollo del concepto (Bárcenas et al., 2016; Singh et $a l ., 2018)$. En general, para el caso de caninos y felinos, son pocos los casos de alteraciones congénitas descritos en la literatura, y se conoce poco sobre la epidemiología de este tipo de afecciones (Molina et al., 2012).

La etiología precisa y la patogenia de SR son desconocidos y puede relacionarse con factores genéticos, mecánicos, endocrinos, metabólicos, nutricionales e in- fecciosos (Ozalp1, 2011; Mateo y Camón, 2008; Leal et al., 2017). Algunos estudios en bovinos sugieren que la SR se hereda de forma autosómica recesiva en esta especie; sin embargo, se necesitan estudios adicionales para confirmarlo (Citek, 2012), mientras que en gatos se cree que puede estar asociado con defectos cromosómicos que incluyen roturas de cromátidas y cromosomas, figuras de intercambio, emparejamientos no homólogos, pulverización y fragmentos dicéntricos, ocasionados por teratógenos ambientales o por factores hereditarios (Ozalp1, 2011).

El SR en bovinos se ha definido como un síndrome de defectos con componentes constantes y variables (Laughton et al., 2005). Generalmente se considera que las características que definen los casos de SR incluyen inversión (retroflexión) extrema de la columna vertebral, eventración de las vís- 
ceras abdominales debido a una fisura de la pared abdominal ventral, anquilosis de las extremidades e hipoplasia pulmonar y diafragmática (Batra et al., 2015). Los componentes variables de la SR incluyen escoliosis, esternón hendido, exposición de vísceras torácicas y anomalías de los sistemas digestivo y urogenital (Laughton et al., 2005; Batra et al., 2015). Los hallazgos descritos en gatos han sido escoliosis de la región toraco-lumbar con desviación derecha, lordosis lumbo sacra, presencia de una fisura abdominal que permite la evisceración de los intestinos delgado y grueso, estómago y bazo, e hipoplasia hepática, pulmonar y cardiaca (Kawata y Tosiro, 1961; Mateo y Camón, 2008, Ozalp1, 2011).

Según Laughton et al. (2005), solo los casos que muestran tanto las vísceras expuestas como la inversión espinal se consideran verdadero SR; sin embargo, los reportes de literatura muestras características muy variables sobre el grado de exposición visceral y de inversión espinal (Laughton et al., 2005), lo que ha permitido que casos en felinos en los cuales no se presenta anquilosis de las extremidades (Lankton et al., 2014) o en los que la lordosis no es tan extrema como para afrontar el cráneo con el sacro (Ozalp1, 2011), características identificadas como componentes constantes de este síndrome descritos para la especie bovina (Laughton et al., 2005), sean reportados como casos de SR verdadero. En este caso no se encontró el componente de artrogriposis y la desviación de la columna vertebral tipo lordosis no fue tan severa como se ha descrito para la especie bovina.

Existen algunos diagnósticos que podrían considerarse como diferenciales en esta patología como lo serían la hernia abdominal, el onfalocele y la gastroschisis, que se descartan en este caso. En el onfalocele los intestinos sobresalen a través de la porción proximal de la apertura umbilical cubiertos solo por una membrana trasparente; en la hernia umbilical hay exteriorización de las asas intestinales a través de la cavidad abdo- minal, pero se encuentran cubiertas por la piel (Makris e tal., 2009; Lucas et al., 2016) y la gastroschisis se describe como un defecto de la pared ventral que en el caso de la especie humana está primordialmente ubicado hacia el lado derecho del ombligo y que es caracterizado por una protuberancia de los intestinos y, ocasionalmente, otros contenidos abdominales no cubiertos por amnios (Sadler, 2010).

Debido a las alteraciones morfológicas presentes en SR, especialmente las relacionadas con la columna vertebral, este síndrome es causa de distocia fetal en rumiantes, siendo requerida con frecuencia su extracción por cesárea; sin embargo, en caninos y felinos la distocia no se relaciona comúnmente con esta patología (Leal et al., 2017). En este reporte el motivo de consulta inicial fue distocia, pero esta no estuvo relacionada con causas fetales y al parecer se debió a causas maternas.

\section{Literatura Citada}

1. Ahuja AK, Singh H, Singh AK. 2017. Fetotomy of Schistosoma reflexus and brachygnathist buffalo calf: a case report. Res J Chem Environ Sci 5: 130-132.

2. Bárcenas Ibarra A, Rojas Lleonart I, Lozano Guzman RI. 2016. Schistosomus reflexus syndrome in Olive Ridley Sea Turtles (Lepidochelys olivacea). Vet Pathol 1: 171-177. doi: 10.1177/ 0300985816651682

3. Batra K, Tewari A, Chandolia R. 2015. Incidence of fetal monstrosities in India: a review. Theriogenology Insight 5: 219229. doi: $10.5958 / 2277-3371.2015$.00024.8

4. Citek J. 2012. Pedigree analysis of Czech Holstein calves with schistosoma reflexum. Acta Vet Scand 54: 22-27. doi: 10.1186/1751-0147-54-22

5. Ferreira D, Santarosa B, Monteiro Toma C, Belotta A, Chiacchio S, Machado V, Gonçalves RC, et al. 2013. Estudo anatomorfológico, radiográfico e tomográfico de Schistosomus reflexus 
em ovino da raça Dorper: relato de caso. Arq Bras Med Vet Zootec 65: 1096-1102. doi: 10.1590/S0102-09352013000400022

6. Gómez Velásquez CA, Pinilla Peña $G A .2018$. Schistosomus reflexus en un feto bovino en Colombia: reporte de caso. Rev Inv Vet Perú 29: 1571-1574. doi: 10.15381/rivep,v29i4.14248

7. Ozalp1 GR, Celikler S, Simsek G, Ozyigit MO, Inan S. 2011. A case of Schistosoma reflexum in a cat with chromosomal aberrations. Reprod Dom Anim 46: 373-376. doi: 10.1111/j.14390531.2010.01649.x

8. Jana D, Jana M. 2013. Studies on Schistosomus reflexus in indigenous cattle in tropical west Bengal, India. Explor Anim Med Res 3: 74-77.

9. Kawata K, Tosiro T. 1961. A rare case of Schistosomus reflexus in the cat. Jap Vet Res 9: 179-181.

10. Lankton JS, Vanderhart DJ, Terrell SP. 2014. Schistosomus reflexus-like malformation in a southern white rhinoceros (Ceratotherium simum simum). J Zoo Wildl Med 45: 708-711. doi: 10.1638/2013-0272R1.1

11. Laughton $K W$, Fisher $K R$, Halina $W G$ Partlow GD. 2005. Schistosomus reflexus syndrome: a heritable defect in ruminants. Anat Histol Embryol 34: 312-318. doi: 10.1111/j.1439-0264.2005.-00624x

12. Leal Bertolo PH, Bastos Andrade Moutinho da Conceição ME, Valente Miranda de Aguirra LR, Martinelli Martins D, Costa de Macedo B, Nassar Coutinho L, et al. 2017. Schistosomus reflexus in a dog and a cat. Acta Sci Vet 45(Supl 1): 235.
13. Lucas F, Panelli E, Nardin Neto E, Kandrotas A, Mastrocinque S, Lataro R, Oliveira G. 2016. Partial hepatectomy in a calf with an omphalocele: case report. Arq Bras Med Vet Zootec 68: 1581-1585. doi: 10.1590/1678-4162-8877

14. Makris SL, Solomon HM, Clark R, Shiota K, Barbellion S, Buschmann J, Ema M, et al. 2009. Terminology of developmental abnormalities in common laboratory mammals (Version 2). Birth Defects Res B Dev Reprod Toxicol 86: 227-327. doi: 10.1002/bdrb.20200

15. Mateo I, Camón J. 2008. Schistosoma reflexum in a cat: insights into aetiopathogenesis. J Feline Med Surg 10: 376-379. doi: 10.1016/j.jfms.2007.12.010

16. Molina VM, Oviedo CA, Casado A, Arias MP. 2012. Schistosomus reflexus en un canino: reporte caso. Rev Med Vet Zoot 59: 50-56.

17. Prestes NC, Megid J. 2010. Uma forma rara de ocorrência do Schistosomus reflexus em bovino. Relato de caso. Vet Zootec 17: 214-218.

18. Sadler TW. 2010. The embryologic origin of ventral body wall defects. Semin Pediatr Surg 19: 209-214. doi: 10.1053/j.sempedsurg.2010.03.006

19. Singh N, Dhindsa S, Singh R, Singh N. 2018. Schistosomus reflexus accompanied by left brachium Amelia and contracture of remaining limbs in a crossbred cattle calf. J Entomol Zoo Studies 6: 1277-1279.

20. Tsuma V, Abuom TO. 2008. A case report of Schistosomus reflexus in a lamb. Kenya Vet 32: 41-43. 